\title{
Multisensory Immersive Analytics
}

McCormack, Jon; Roberts, Jonathan C.; Bach, Benjamin; Freitas, Carla Dal Sasso; Itoh, Takayuki; Hurter, Christophe; Marriott, Kim

\section{Immersive Analytics}

Published: 18/10/2018

Publisher's PDF, also known as Version of record

Cyswllt i'r cyhoeddiad / Link to publication

Dyfyniad o'r fersiwn a gyhoeddwyd / Citation for published version (APA):

McCormack, J., Roberts, J. C., Bach, B., Freitas, C. D. S., Itoh, T., Hurter, C., \& Marriott, K. (2018). Multisensory Immersive Analytics. In K. Marriott, F. Schreiber, T. Dwyer, K. Klein, N. Henry Riche, T. Itoh, W. S., \& B. H. Thomas (Eds.), Immersive Analytics (1 ed., Vol. 11190, pp. 57-94). (LNCS). Springer Nature.

\footnotetext{
Hawliau Cyffredinol / General rights

Copyright and moral rights for the publications made accessible in the public portal are retained by the authors and/or other copyright owners and it is a condition of accessing publications that users recognise and abide by the legal requirements associated with these rights.

- Users may download and print one copy of any publication from the public portal for the purpose of private study or research.

- You may not further distribute the material or use it for any profit-making activity or commercial gain

- You may freely distribute the URL identifying the publication in the public portal ?
}

Take down policy

If you believe that this document breaches copyright please contact us providing details, and we will remove access to the work immediately and investigate your claim. 


\title{
3. Multisensory Immersive Analytics
}

\author{
Jon McCormack ${ }^{1}$, Jonathan C. Roberts ${ }^{2}$, Benjamin Bach ${ }^{3}$, \\ Carla Dal Sasso Freitas ${ }^{4}$, Takayuki Itoh ${ }^{5}$, Christophe Hurter ${ }^{6}$, and Kim \\ Marriott $^{7}$ \\ 1 Monash University, Australia \\ Jon.McCormack@monash.edu \\ 2 Bangor University, UK \\ j.c.roberts@bangor.ac.uk \\ 3 University of Edinburgh \\ benj.bach@gmail.com, \\ 4 Federal University of Rio Grande do Sul \\ carla@inf.ufrgs.br \\ 5 Ochanomizu University, Japan \\ itot@is.ocha.ac.jp \\ ${ }^{6}$ Ecole Nationale de l'Aviation Civile (ENAC), France \\ christophe.hurter@enac.fr \\ 7 Monash University \\ Kim.Marriott@monash.edu
}

\begin{abstract}
While visual cues are traditionally used for visual analytics, multimodal interaction technologies offer many new possibilities. This chapter explores the opportunities and challenges for developers and users to utilize and represent data through non-visual sensory channels to help them understand and interact with data. Users are able to experience data in new ways: variables from complex datasets can be conveyed through different senses; presentations are more accessible to people with vision impairment and can be personalized to specific user needs; interactions can involve multiple senses to provide natural and transparent methods. All these techniques enable users to obtain a better understanding of the underlying information. While the emphasis of this chapter is towards non-visual immersive analytics, we include a discussion on how visual presentations are integrated with different modalities, and the opportunities of mixing several sensory signals, including the visual domain.
\end{abstract}

Keywords: immersive visual analytics, multisensory visualization, haptic data visualization

\subsection{Introduction}

We live in a world that excites all our senses. When walking down the corridor the clatter of our footsteps changes as the corridor ends and the stairwell starts. As a person in this workplace, we understand that we have moved from one location to another. The feeling of the roughness of our feet on the stairs, as we 
walk down each step, gives us feedback that we are walking downstairs and helps us to stand upright rather than falling down at each step. We feel the change in pressure on our muscles as we walk, and the smoothness of the wooden hand-rail as we balance our steps. We can hear the sounds of our colleagues talking at the bottom of the stairs, long before we can see them. Furthermore, we readily realize that a colleague has previously walked along the same way because we can smell their perfume in the air. We become immersed in these surroundings. We see, hear, touch and even smell aspects of the building. All these different sensory cues help us to understand where we are, that we are going in the right direction (down the stairs towards our colleagues talking) and that we are able to understand data from the environment (such as guessing how many colleagues are downstairs, just by hearing them). We are certainly immersed in this world.

In immersive analytics we wish to achieve the same result. We want to be immersed in data in such a way that we can perceive all the nuances of the underlying information. Not only should it therefore be possible to visually see our data but to hear, touch and smell the data as well. Furthermore, we would be able to interact through forces, select through gestures or zoom by just by moving our body.

Using all our senses to perceive and interact with the information affords new possibilities. Interfaces can be built that are more natural, which match well to the day-to-day movements of our bodies. We have opportunities to display substantial quantities of data, and use human metaphors such as front and back (where interesting aspects are in front of us, with those items of less interest pushed behind us). We can also include more users, and perform collaborative tasks with them. For example, tangible objects can be used with virtual displays with the implicit notion that whoever holds the object can speak and express their views on the data that is being displayed.

As authors of this chapter, we're excited about the potential and possibilities of this new frontier in immersive analytics. Navigating the research across multiple senses and modalities is complex and difficult. So we organise the material in this chapter by the five main senses: Vision, Sound, Haptics (touch), Smell and Taste.

Using vision (Section 3.4.) to understand data has been extensively studied (e.g., $[18,84,117]$ ), and consequently it would be possible to create (another) whole book about visualization. This is obviously not our goal. Issues of spatially immersive visualization are covered in great detail in Chapter 2. Here, we review the most important concepts relevant to multisensory immersion.

Sound (Section 3.5.) includes abstract sounds and audible signals, as well as spoken words (auralization) and forms of spatialised sound.

Haptics (Section 3.6.), especially tactile interactions enable the user to feel different textures on their hands and body. Kinesthetic interaction deals with issues of muscle movement and position. We are able to feel and exert forces with our arms and feet.In fact, haptic devices can also simultaneously be actuators, providing both input and output. 
Smell and Taste (Section 3.7.) are covered in the same section, not only because the semiochemistry is linked, but also there are fewer designs of data visualization and interaction in this category.

There are many ways to design these systems, and definitely many issues when combining multiple senses together. In Section 3.8. we explore how the user may benefit when the same data is displayed through different senses as complementary presentations. We explore issues of technology and capability of humans to comprehend large quantities of data. We conclude in Section 3.9. with a discussion of future work and directions for multisensory immersive analytics.

\subsection{Multisensory Presentation and Analysis}

Looking at prior research it can be observed that developers have created numerous multisensory interactive devices. For instance, fun-rides at a fair or resort, excite all our senses, and furthermore there are often rides that use three-dimensional screens, haptic feedback and high-quality sound.

Indeed, it is clear that multisensory storytelling and multisensory visual presentation has been explored in the past. An excellent early example is Morton Heilig's Sensorama system (see [100]). Sensorama placed the user in a multisensory environment allowing them to smell, hear stereo sound, feel vibrations of the seat, and feel the wind in their hair: in combination creating the illusion of presence within another world. Even since 1962 (when Sensorama was patented) researchers have investigated different technologies to display data through multiple senses. However, recently we have seen a step-change in the engineering capability of virtual and augmented reality technologies. Sensors and actuators are smaller, lighter, cheaper, more readily available and easily to connect with each other. Generic, low-power micro-controllers and micro-computers (such as Arduino, Raspberry Pi or BeagleBone) have become small and light, which enable developers to quickly build demonstration interfaces that investigate a variety of multisensory techniques and novel ideas, and companies have produced many excellent commercial devices (such as Oculas Rift, Microsoft HoloLens, HTC Vive, Nintendo Wii).

Any modern computer or digital device employs multisensory interaction: visual displays, touch screens, stereo or multichannel sound and haptic feedback are commonly found on many everyday devices.

The information visualization field has also matured. Researchers have discovered ways to display large abstract datasets, either by using large displays (e.g., $[10,60,102]$ or employing some dimensionality reduction method $[28,31]$ that allows for large data sets to be displayed in regular-sized or even small screens. Visual analytics interfaces have been developed that adapt parameters for the underlying algorithms. Machine learning, data mining, filtering, statistical and other analytic algorithms can all be run interactively. The visual mappings and the underpinning analytics functions are being integrated, such to provide the user with powerful exploratory and intelligent analytic tools. While these systems are extremely useful, they are not spatially immersive in the sense that 
the user feels like they are located in a virtual "data world" and for the most part they do not utilize or excite all the human senses, only vision.

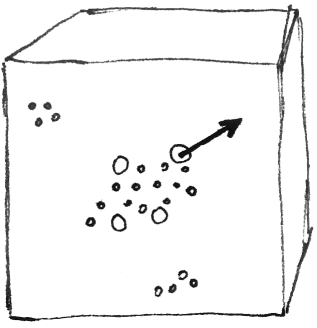

Select one point

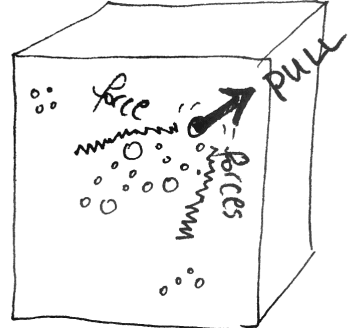

haptic feed back to pull point away

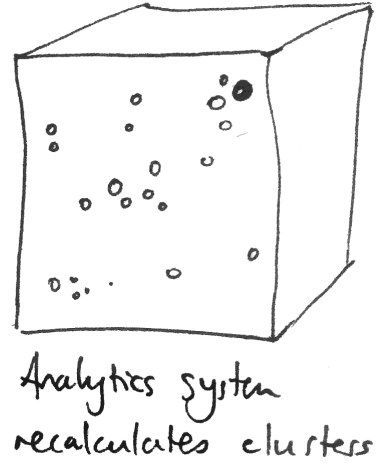

Fig. 1: In this conceptual example, the user is immersed in a large scatterplot. They can select one or more points, move them away (feeling forces related to their closeness to the rest of the points) and place them in a new position, where upon the analytic grouping re-calculates and displays the new clusters.

This chapter explores possibilities and potential design challenges for multisensory immersive analytics, where the user is placed in the heart of their data, they can interact with the information using touch, their body, or arms (for instance), and the data is presented through multisensory stimuli. To motivate this research, let us consider three illustrative examples.

The first is an imaginary example where the user is placed inside a threedimensional scatterplot of their data, see Figure 1. We could imagine that the scatterplot represents an international company's credit card transactions over one year. The user wishes to investigate how fraudulent transactions spread over the globe. The scatterplot itself would be a multi-dimensional scaling of many variables, with color presenting the cost of a transaction. An immersed user would be able to stand inside the plot, surrounded by billions of data points. They could move their hands through the data, like moving their hands through water, to push some of the points around. Spatialised sound could be used to highlight potential fraud occurrences and draw the user's attention to particular locations, even those outside the current field of view. They could select a group of points with their hand, and start to move them away, with the idea of moving them closer to another group. Forces could be mapped onto clusters to keep them together in one unit, but the user could temporarily pull clusters away to explore them, which snaps them back to their original position when they have completed their operation. In another interaction style, the user could group points together, gesture to move them to a new position, at which point an analytic algorithm re-evaluates the statistics and a new dimension-reduction 
result is displayed back to the user. This dynamic interaction enables the user to be immersed in a multisensory environment: to see, hear and feel the data in a spatial datasphere.

The second example relates to air traffic controllers and the emerging usage of remote towers. Thanks to technological improvements, live video stream of an air field can be broadcast to remote site where air traffic controller can monitor and regulate aircraft movements. In this specific example, it is important to provide sufficient information so that the immersed user can have a suitable situational awareness. Remote towers encompass a multisensory virtual environment with the visualization of the air field, haptic feedback, audio and multimodal interaction systems [26].

Our third example of multisensory analytics are situations when data analytics is required but vision cannot be employed for this purpose. The most obvious reason for this is that the analyst is blind or has severe vision-impairment but other reasons might be that the environment is dark such as when soldiers are patrolling at night ${ }^{1}$ or that vision is required for another purpose. There has been considerable research into how sound and touch can be used by people with severe vision impairment to understand data. For example, sonification of line charts and bar charts (see Section 3.5.) or haptic presentation of spatial and network data (see Sections 3.6. and 3.6.4.). In the near future one can imagine using interactive data physicalisation augmented with audio feedback to provide an immersive interactive data exploration tool for data scientists with severe vision impairment.

As our three examples illustrate, multisensory approaches can enrich the fidelity of our experiences with data analytics, making data more accessible, our experience of it potentially more memorable, and less cognitively challenging in critical situations.

\subsection{A Framework for Multisensorial Immersive Analytics}

Figure 2 provides a schematic drawing of a design framework for multisensory immersive analytics. The framework aims to help users understand their data better by providing a multisensory environment that utilises traditional visualization (data visualization), sound (sonification), touch (haptification) and even smell (olfaction) or taste (gustation) to immerse the user in their data. As the visual sense is certainly the most explored and used in current analytics systems, through data visualizations, we start by recapitulating the most important concepts from visualization.

In traditional data visualization data elements and their attributes are mapped to geometric elements and their visual and spatial attributes [84]. The rules dictating how the data is mapped to the visual variables is called the visual

${ }^{1}$ Braille is based on a tactile writing system invented by Charles Barbier for the French army so as to allow soldiers to safely communicate during the night. 


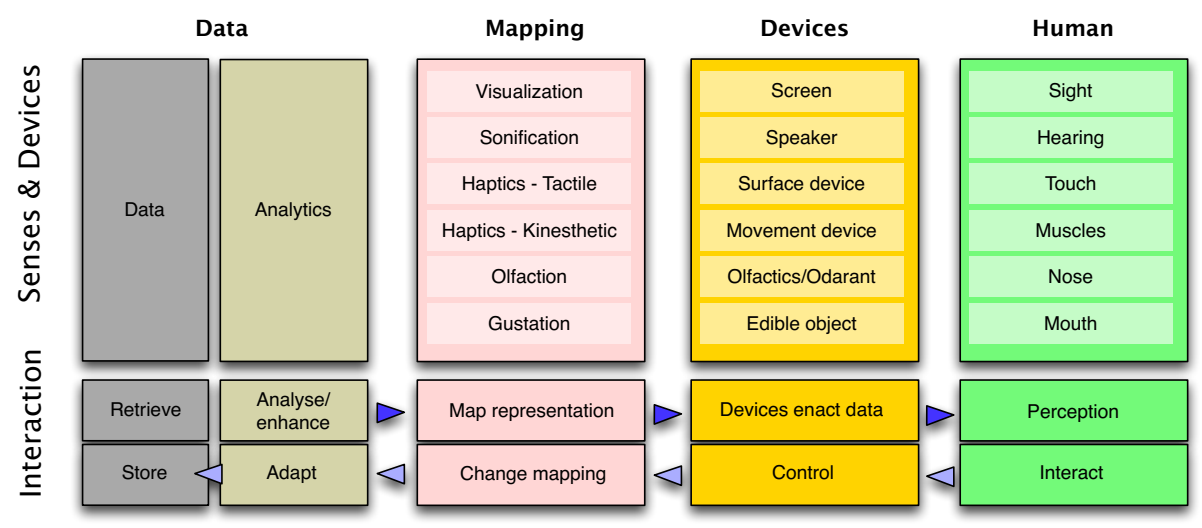

Fig. 2: The design framework for multisensory immersive analytics. Data is mapped onto sensorial channels in the different sensory channels and presented using a variety of devices to the corresponding human sensory system.

mapping (or visual encoding). For example, a visual mapping might map each car in a data set to a filled circle and the car's gas consumption to the area of the circle. The resulting picture is the visual representation (or visualization) of the data.

Multisensorial immersive analytics generalises this to other senses. We define a sensorial mapping as a mapping from data elements and data attributes to sensory channels (sight, hearing, touch, proprioception, smell and taste) and their respective sensorial variables (color, pitch, roughness, etc.). This sensorial mapping is then rendered perceivable through different kinds of devices that then stimulate the human senses (Figure 2). In principle, any value can be mapped onto any sensory channel and sensorial variable. For example, a larger value can be mapped onto a darker color, a louder sound, or faster vibration on a vibro-tactile device.

However, because of the characteristics of the human sensory system or limitations of the display device some mappings will be more effective than others, as shown for visualization in the work by Mackinlay [74]. By this we mean that the chosen sensorial variables are more effective in their mission to allow the user to discriminate and compare data values. For example, an effective sensorial mapping would map four categories of elements to four clearly distinctive colors such as red, blue, bright-orange, and green. An ineffective mapping would map the four categories to four very similar colors. Similarly, an ineffective mapping would be to map, for example, dozen values to dozen very different colors, as the human visual system may be incapable of memorizing and clearly decoding a large number of different colors $[92,117]$.

The data is not simply presented to the user passively: the user can actively interact and explore it. Not only does the user wish to see, feel and hear the data, but they also wish to interact with it. Interaction is two-way. It is clear, that some 
modalities are readily input/output. The movement of our arms (kinaesthetic forces) act both as input devices and output devices. We can hear data through our ears (sonification), but we use our mouth (or maybe our body) to create sounds. Different senses therefore afford different types of representation and interaction.

We represent this two-way interaction in Figure 2 by arrows. In particular, this is one of the areas where immersive analytics needs much research. For instance, it is not clear (for a given task) how the data should be mapped into (say) the visual channel, or how a user could interact with the data haptically (for instance), or then what type of interaction method (menu, gesture, etc.) is used to control the functions to alter the data analytics. Within this system the user is immersed as a human-in-the loop. We encourage the reader to refer to Chapters 4 and 5 for more information about interactive systems and human-in-the-loop analytics.

In the remainder of this chapter we will look at the different sensory channels and variables and how they might be used to understand data. In order to do this it is useful to consider the kinds of dataset that the user might be interested in exploring [84]. These include: tabular data, which is conceptually organised into a table with each row corresponding to a different data point or item, and each column corresponding to a different data attribute; network data consisting of nodes (or items) and links between these nodes representing different kinds of abstract relationships; spatial data in which items are associated with a geographic location or region, and this geographic key is a natural way for organising and understanding the data; field datasets that are sampled from a continuous, conceptually infinite domain and textual datasets.

Attributes in data items are values that can be measured or logged. They can be [9] categorical or ordered. Categorical data does not have an inherent ordering. The nationality of people is categorical. Moreover, categorical data is often organised into a hierarchy. An example would be the rank of a specimen in the taxonomy tree of living species. Ordered data has a natural ranking of elements. It has two subtypes: ordinal and quantitative. Ordinal data can be ranked but the difference between items does not make arithmetic sense, for instance in degrees of preference. An example would be names. For quantitative data differences in value can be compared, e. g., height or weight. The respective data type of attributes can have implications on the sensorial mapping [9].

Furthermore, the analyst may not only interested in visualising the raw data. They may also wish to transform and generate new measures from it. For instance they may wish to simplify, aggregate, arrange, re-arrange, average, calculate and display a range of the data, and so on.

\subsection{Visual Presentation}

Human vision is the most investigated channel for data presentation, e. g., [18, 84, 117] to name but a few. This section summarizes the most important findings for 
data visualization in order to provide a benchmark for the design and discussion of data presentation using other sensorial channels in sections that follow.

\subsubsection{Physiological Characteristics of Vision}

Vision is the main sense for perceiving our environment. It has evolved to allow us to quickly build a 3D model of the objects in our environment from the essentially $2 \mathrm{D}$ projection of the environment on our eyes. The visual system has sensors that respond to light. It has a wide area of perception-approximately $100^{\circ}$ vertical and $200^{\circ}$ horizontal-that provides parallel information acquisition in a continuous flow as well as a narrow focus of attention frame (the fovea) which can provide detailed information [117]. It does not need to have physical contact with objects to acquire this information.

The visual system has three main levels or stages [117]: (1) Parallel processing to extract low level properties: color, texture, lines and movement; (2) Rapid serial processing divides the visual field into regions of similar color or texture and achieves proto-object recognition of surfaces, boundaries and relative depth. This is driven both top-down by visual attention and bottom-up by low level properties and (3) visual working memory: object recognition and attention, this is under conscious control.

Pre-attentiveness: Low-level visual processing occurs pre-attentively and in parallel. This means that visual encodings of data do not have to be consciously recognised by the user. Instead a user can perceive a piece of information before any conscious cognitive activity has happened. Thus red objects "pop out" from blue objects.

Gestalt Theory: Much work on perception of graphic representations is based on Gestalt Theory [118]. This investigated the basic perceptual laws that the human brain uses to pre-attentively group graphic elements as part of protoobject recognition. Elements are grouped by proximity, similarity (e. g., same color or shape), closure and common region, explicit connection (e.g., lines connecting points), continuity and common fate [117].

Depth cues: The visual system uses a wide variety of depth cues to infer the shape of $3 \mathrm{D}$ objects from the essentially $2 \mathrm{D}$ images falling on the eye. Depth cues include occlusion, linear perspective, changes due to motion of the viewer or object, blurriness due to accommodation, convergence and binocular disparity. See Chapter 2 for more details.

\subsubsection{Visual Elements, Variables and Idioms}

The visual system allows a rich variety of visual elements and visual variables to be employed for representing different aspects of data. Jaques Bertin [9] identified three kinds of visual elements: points, lines, and areas. More recent literature includes surfaces and volumes in graphical 3D space [78]. Attributes of data elements can be conveyed by visual variables applied to these visual elements. Bertin [9] identified seven visual variables location, size, color, opacity, orientation, texture, and shape. More recently the use of digital and dynamic 
computer displays has allowed Bertin's initial set of visual variables to be extended by containment, volume, slope etc. [74] and variables of motion (flicker frequence, direction of movement, rythm, onset, etc.) [19,63].

A large number of studies $[24,32]$ have investigated the comparative effectiveness of these visual variables for different kinds of data representation. The results are summarised in $[78,84]$. For instance, in order of decreasing effectiveness, size, color, motion and shape can be used to show categorical attributes while linear position, length, angle, area, depth, color and curvature and volume can be used to show ordinal attributes. Due to several studies, it is already well known which visual variables can be distinguished pre-attentively and their separability, i. e., how much different variables interfere with each other in pre-attentive processing [117].

Over the last five hundred years a wide variety of ingenious visual idioms have been invented to show different kinds of data with these visual elements and variables. For instance, bar charts, scatter plots and line charts all use position but with different visual elements to show different kinds of tabular data, choropleth and other kinds of data maps show spatial data, while nodelink diagrams and adjacency matrices show network data. These components: visual elements, visual variables and visual idioms a provide a basic structure for understanding, describing, and creating visualizations. It also allows - to some extent - evaluation of the perceptual effectiveness of a visualization. However, assessing the real value of any visualization is still complex.

\subsubsection{Presentation Technology}

Emerging presentation technologies for VR and AR are discussed more fully in Chapter 2. For our purposes what is interesting about this technology is that as well as providing immersive visualization it routinely provides stereo or even surround audio and haptic feedback is becoming increasingly common. For instance, the controllers for the HTC Vive or Oculus Rift incorporate haptic feedback and the Nintendo Switch Joy-Con controllers have multiple haptic motors that considerably enhance the sense of immersion when used in games.

\subsubsection{Vision in Immersive Analytics}

Vision is the basis for visual analytics and will undoubtedly remain the primary sensory channel in most immersive analytics applications apart from those in which for some reason vision cannot be employed. The reasons for this are that vision provides multiple sensorial variables, high bandwidth and parallel preattentive processing. There are well established frameworks and tools for creating effective visualizations as well as commodity technologies for presentation.

\subsection{Sonification and Auralization}

Like vision, hearing is a highly immersive and important sense, often considered the second most important after vision. Humans can become highly sensitive to 
different nuances in sound through training, prominent examples being visually impaired persons and musicians. With specialised training it is even possible to use sound and hearing as a form of echolocation: to understand oneself spatially in an environment and successfully navigate it without vision [64].

\subsubsection{Perceptual Characteristics of Sound}

Sound is the movement of vibrational energy through a physical medium (typically air, but sound can also be heard underwater, for example). By sound, we are actually referring to a collection of psychophysical aspects. The three most prominent being loudness, pitch and timbre. A young, healthy human adult can detect pitches with frequencies ranging from $20 \mathrm{~Hz}$ to $20 \mathrm{kHz}$. As frequencies drop below $20 \mathrm{~Hz}$ the sound perception transitions to rhythmic sensation, as each sound pressure peak can be individually resolved. Sounds can be sensed as quietly as $-20 \mathrm{~dB}$ and are perceived as pain above $140 \mathrm{~dB}$. The ear is more sensitive at certain frequencies than others. Sound is perceived best between 1 and $4 \mathrm{kHz}$ (the characteristic frequencies of human speech). The smallest noticeable difference (or just noticeable distance, JND) between two tones (pitches) is typically 5-6 cents, 1 cent being 1/100 of the distance between two semitones with 12 semitones in one octave. Timbre (sometimes referred to as tone color) is our identification of the frequency characteristics of a specific sound. For example, a violin and trumpet can play the same pitch, but sound different due to the different levels of harmonics from the fundamental frequency of the note. Moreover, perceived sound can consist of multiple individual sources (such as a symphony orchestra), as well as varying overtones. Together, all these characteristics can produce a unique and distinguishable "sound image", similar to a picture, visual object, or visual glyph.

In addition to recognising loudness, pitch and timbre, humans are also able to recognise rhythmic patterns and to spatially locate the source of a sound in space, including sounds that come from behind or above (although not with the same accuracy uniformly around the body). Spatial location comes predominantly from the sound source arriving at different times in each ear and from cues due to reflections off surfaces in the listening environment. Analogous to stereo vision, good sensing of a sound's location is dependent on having two functioning ears.

\subsubsection{Comparison to Vision}

In fact, sound has many analogous perceptual characteristics to vision. It can be processed in parallel (multiple sounds at the same time) and position cues with gestalt rules including similarity common fate and proximity are used to build up a 3D model of the objects in the environment [34]. Like vision there is no need to have contact with objects to sense them. Often, the same terms are used to describe characteristics of visual stimuli and aural stimuli; loud, salient, warm, contrast, blur etc. (A fact which holds for other senses as well, e.g., the tactile senses.) Finally, sound is omnipresent meaning that the user does not have to focus his/her attention on it (similar to vision, for example) but also that 
he/she cannot easily escape a perception. Constant, low-level sounds rapidly fade from conscious awareness (e.g., background sounds like office air conditioning or background traffic noise), so one needs to be careful when using them in an immersive analytics application. Similarly, loud fluctuating sounds immediately attract attention, but cause fatigue or even distress if people are exposed to them for too long.

\subsubsection{Perceiving Data with Sound}

Communicating information with sound is very common, even though often we don't think of it as "information" in a traditional sense: medical monitoring instruments, sonar, Geiger counters, bell tower clocks with different bells for hours and quarter hours. Similar to visual mappings, those "instruments" map (encode) characteristics of the data or information to characteristics of the sound (frequency/pitch, volume, timbre, localization, etc). Such a mapping can be called sonification [46, 47,69]. For example, low pitched values can be mapped to low quantitative values in the data and higher pitches to higher values.

Examples of Data Sonification Many existing data sonifications are inspired by those in the visual domain, such as Franklin and Roberts' pie chart sonification [36], Dingler and Brewster's AudioFeeds [30] which spatialises social network data in a soundscape that surrounds the user, or Saue's [104] work to present a general model for sonification of large spatial datasets. Further examples of existing sonifications include an interactive sonification of multidimensional data where users can change parameters of the data-to-sound mapping while listening [4]; the "Iraq body count" explores the relationship between deaths in Iraq and the oil price through sound [110]. Finally, Xi et al. present a tool for the sonification of general time series data [121]. Further examples can be found in [73]. Conferences such as the International Conference on Auditory Display specifically focus on research regarding the sonification of data from technical, aesthetic and psychological perspectives.

Scanetti et al. [105] discussed the merits of using sound to represent meaning in data, introducing their own sonification system. Madhyastha et al. [76] summarized sound attributes for data sonification and then introduced an example sonification toolkit. A study by Flowers et al. [33] showed equivalent performance for users interpreting scatterplots visually, and through a mapping to sound. More topics and issues on sonification are introduced in a book [46] and a survey paper [68].

As already mentioned, sound can be used in situations where no visual display is present or a visual display is ineffective or distracting (e.g., Geiger counter). Sound is indirect, i.e., a user is free to look and move where they want. Sound can be present in the background for monitoring purposes (although as discussed above, continuous sounds with little variation quickly fade from conscious perception), and come into attention in critical moments via changes in perceptible characteristics. Sound can further be used to enhance another 
modality; for example, Baum et al. [7] discuss how immersive sound can be used within a CAVE environment, and Hoggan and Brewster [49] studied the mix of audio and tactile feedback in information visualization on a mobile touch screen.

Sound is also effective as a cue to notify variation of data. Madhyastha et al. [76] categorizes acoustic attributes effectively applied to sonification as follows:

Pitch: One of the most intuitive ways to express relative magnitude. Mapping to musical notes is more likely to form human-recognizable patterns rather than mapping to arbitrary frequencies (note that frequency and pitch are related logarithmically).

Loudness: Useful to attract attention. Needs to be used carefully as different pitch or timbre may cause differently perceived loudness.

Timbre: Useful to draw distinctions among multiple data categories and many tools allow the choice of voices or tones from predefined sets.

Location: Effectively used only to categorize several discrete values, because precise perception of the elevation of sound source location is relatively poor.

Musical components: Effective to represent particular patterns. For example, change of rhythm patterns can notify temporal separation of information. Melody is a good associative element that allows users to remember associations.

\subsubsection{Designing with Sound}

One of the important applications of sonification has been for users who are blind or partially sighted. For example, Brewster and his colleagues have explored the use of speech and non-speech audio for presenting tabular data to people with vision impairment $[15,94]$ while Zhao et al. [124] investigated the presentation of maps and tabular data.

However, despite initial research on the perception of auditory variables (e.g., $[33,40])$, design guidelines and practices about how to build "auditory displays" for data analysis, are still lacking [69]. Ludovico's sonification space [73] and their differentiation into sonic plots (parametric auditory mapping of data to sonic characteristics), system-state description (no precise parameter mapping), auditory support of interactive exploration tasks such as navigation, or in augmented reality setups, are a first step towards a more general framework similar to the one in visualization. Indeed, several concepts [73] are already similar to visualization: orthogonality of auditory variables [67], perceptibility and the number of distinct values (of pitch, volume, etc.) that humans are able to perceive [88], and redundant encoding [66]. Furthermore, as Ludovico et al. further point out, "many symbolic aspects of sound are culture-dependent, so it is difficult to create a sonification having a universally accepted meaning".

Sound, music and voice are also an important narrative devices, as becomes immediately apparent when trying to watch a cinematic film with the sound turned off. In cinema for example, musical scores serve many purposes beyond just aural aesthetics. These purposes include: the anticipation of future events, 
the arrival or return of a character, reinforcement of visual movement or action, reflection on past events, even action or events that occur off screen.

\subsection{Haptic Displays}

The sense of touch is important to human beings. As we move our muscles we gain an understanding (proprioception) of our body orientation. As we grab a cup we can ascertain (through touch) what type of material the cup is made from, how hot the drink is inside, and how much force we need to hold it. As we pick up a shirt we feel the properties of the fabric, its texture and silkiness, and might be able to understand its composition. We can also feel its weight. All this sensory input helps us to build up a mental model of the object and make deductions about the quality of the garment we are holding. We understand the world, not only through sight, but actively through our sense of touch.

The word haptics comes from the Greek word haptikós, which literally explains our ability to touch or grasp something. We not only can feel the forces exerting on us, but we can engage with the scene and push or pick up the object that we are observing. Therefore, when we perceive characteristics of the object through the sense of touch, haptics is being used as output to us; when we use our forces to interact with objects in the scene, haptics is being used as input to the objects we are interacting with.

\subsubsection{Physiological Characteristics of Haptics}

We sense touch and movement through many interrelated receptors that are located all over our body. Through our experience of touching and manipulating many different objects we build a deep understanding of how various materials react and feel. That is used to decide what type of object we are holding. For example, we know that metals are generally cold and wood is warm to touch. We can also use this sense to perceive changes in the temperature of bodies, for example, to help us figure out whether someone is probably becoming ill, because temperature is increasing along time.

Our body is full of receptor cells that when stimulated send signals along nerve cells to the brain. These network of nerve endings and receptors is known as the somatosensory system (see [93], Chapter 8). Humans can distinguish between brief tactile sensations and continuous touch, and can understand what part of the body a force has been applied and when an stimulus triggers a pain sensation. There are four main types of receptors: mechanoreceptors, thermoreceptors, proprioceptors and nociceptors.

Mechanoreceptors detect indentations and vibrations on the skin. These types of receptors give rise to sensations including pressure, vibrations and texture. The two most sensitive mechanoreceptors are found in the top layers of the skin and on non-hairy parts of the body such as the lips, tongue, palms, or the soles of our feet. Merkel's disks enable the sensation of slowly adapting 
change, whereas Meissner's corpuscles react to rapid change. Together these allow humans to understand how long something has been touched. Along tendons, joints and muscles we find Ruffini and Pacinian corpuscles, which detect vibrations in our bones, stretching of skin and movement of limbs [39].

Thermoreceptors enables humans to perceive the temperature of objects on the skin and are found all over the body [45]. Cold receptors work at lower temperatures, and hot receptors work to sense hotter skin temperatures until the pain receptors take over. Early works on this kind of receptors identified that response to warm and cold temperatures are localized, i. e., separate spots in the skin respond to selectively to temperature. Moreover, there is a continuum between these two sensations: from indifferent - lukewarm - warm - hot - heat pain on the warm side to indifferent - cool - cold - cold pain on the cold side [45].

Proprioceptors help humans understand their own body, by providing continuous and detailed information about the position of limbs and other body parts. These receptors are found in muscles, tendons and joints, and in fact are low-threshold mechanoreceptors specialized for conveying information from the musculoskeletal system. In the case of the head position and movement, proprioceptors are integrated with the vestibular system [93], Chapter 8.

Nociceptors enable humans to perceive pain and help to protect the body from harm. There are different nociceptors that enable to sense excess of mechanical stimuli and temperatures, and chemical substances (such as from an insect sting or various spices). Thus, the other receptors respond from stimuli within certain range; when the stimuli go beyond a certain threshold the nociceptors trigger a signal which is eventually translated as a pain sensation. There are three major classes of nociceptors in the skin: mechanosensitive nociceptors, mechanothermal nociceptors, and polymodal nociceptors. The first two classes are faster-conducting nociceptors that are organized as clusters of sensitive spots and respond selectively to noxiously intense mechanical or thermal stimuli. The polymodal nociceptors tend to respond to thermal, mechanical, and chemical stimuli [93]-Chapter 9.

\subsubsection{Comparison to Vision}

It is well known that vision provides more information from the external world than all the other senses combined [117]. But this also ignores the complexity of understanding our surroundings. Although it is true that our eyes can detect very detailed and complex light changes, each sense should be considered important, complementary yet different. Humans build a picture of the world using all possible sensory input. Our brain will acquire signals from different sensors (including hands and eyes) [113]. In fact, depending on the task, one type of haptic receptor can be more important than the other [13]. For instance, if an object is too hot, we will instantly drop it, and only after fully perceive that the object is hot.

Our eyes are set at the front of our face, separated so that the brain can build a stereo picture, with vision forward-facing. Thus to see something that is located 
behind us we need to physically turn our whole body. As we rotate our body, the proprioceptors in our muscles send signals to our brain to say how much we have moved. In immersive analytics we need to be mindful of the relationships between different senses. For example, imagine you have a large display. There are two options to make the picture bigger: you can either increase the size of the displayed object or get the user to physically move closer [3]. As the person walks they are judging distances and can use this information to understand how large the observed object really is.

We can also consider other advantages in using haptic sensors. First, because the receptors are located over the whole body (rather than just at the front of our head), they could potentially be used to create a better sense of immersion. With an actuated body-suit, for instance, the user would instantly know something in front of them or behind them. Second, it is possible to physically move someone, perhaps on a treadmill or robotic arm. The computer can literally be used to effect or refocus the users' attention to something else. This is similar to leading someone by the hand through a space. Third, we use our arms, hands and fingers to input commands to a computer, and also receive feedback from the computer itself. Our bodies may occasionally perceive lights with our vision passively, but haptics is more bidirectional, because we sense the position of our muscles as well as moving them. It is possible therefore that the computer changes haptically at the same time as the user pushes the device, thus creating a dynamic feedback loop.

Much published research supports the notion that the haptic system is less accurate than the visual system at object identification, or that sight dominates touch. However, many of these supporting papers get the user to manipulate abstract (non familiar) objects, or static raised maps [72] that are meant to be read by a finger. While people may struggle to accurately recognise arbitrary objects, haptics can be very effective to identify familiar objects [61]. For example, in a game to select a specific toy from a bag of general toys, the player will easily (and quickly) put their hand in the bag and pick the right toy. When locating the toy, we use fine motor controls, understand the texture, temperature and roughness of the objects through our fingertips. Current technology is problematic, because most haptic devices only activate one type of receptor. This technological limitation has implications for immersive analytics. Ideally we need to have display technologies that can mimic the intricate and nuanced properties of real objects. Indeed, an important aspect to be considered in artificially generating a sensation that can be used for recognizing some object (or data) is the fact that haptic modalities interact. An interesting study by Rincon-Gonzalez et al. [97] examined the relationships between tactile and proprioceptive modalities. They showed that signals, which would normally be attributed to tactile senses and self-movement, interact both perceptually and physiologically in ways that complicate the understanding of haptic processing. Another finding was that tactile sensation induced on the fingertips can vanish by the changing the posture of the fingers. Although their primary aim was to improve neuroprosthetic systems by investigating the neural processes underlying haptics, they also provided an 
interesting discussion about the difficulties in designing effective information representation through haptics.

\subsubsection{Data Perception and Understanding Through Haptics}

The purpose of immersive analytics is to support the process of obtaining insights from data, i.e., to gain value from the data whatever the form in which it is represented. In other words, the sense that we use (be it vision, touch, or something else) should represent the information and allow us - in the case of haptics - to perceive a force, weight, or temperature, for example, and associate the perceived stimulus with a value. We may be accurate in our sensing and say "the first bar in my bar chart represents a value of $90 \%$ ", or we may be able to ascertain relative values: "the first bar is bigger than the second, so there are clearly more women than men in my class".

A question we should ask ourselves as researchers in immersive analytics is: "how can we effectively display data through the sense of touch?'. In other words, "What haptic variables do we have available?" As previously discussed, in visualization the visual variables [9] include size, position, color (hue, saturation, value) and texture. The haptic variables include force, position, vibration, texture and temperature. To represent value, these cues are adapted. For instance, a larger value in a dataset could be mapped to a higher frequency vibration, greater force, or a hotter temperature. Complex structures can also be communicated as one unit. For instance, in Braille, letters are organised as a unit of raised dots, or a set of vibration, movement and forces could present a haptic glyph [99].

Another question relates to suitable technologies: "What technologies do we have to excite these senses and to allow us (the user) to understand the value of the underlying data?" To answer this question we need to think how we haptically manipulate an object. When we pick up an object in our hands we will move it around, use two or more fingers to judge distances, feel how the object pokes into our skin (to understand how textured the object feels). In our manipulation, we may even pass it from one hand to another. We are not merely picking the object up and holding it still, we are instead actively investigating it with our hands. It is through this motion that we explore the object, exciting a range of receptors, from the slow to the fast response, sensing small textures and the principal corners and edges of an object. Therefore, we can either create static objects and allow the user to explore them through manipulation, or use a computer controlled device to mimic different forces and textures, to excite the receptors on the body. This gives rise to two distinct approaches: data physicalization and haptic data visualization (HDV) or haptification, using haptic devices.

Data Physicalization. In this modality the developer creates a haptic object that presents the data. Static tangible objects are very effective. This is especially relevant for immersive analytics. It is easy and cheap to $3 \mathrm{D}$ print objects from CAD models. These can become digital surrogates, or miniature representations of objects. For example, archaeologists in the field can survey buildings or 
monuments, create 3D models and print 3D digital representations. The heritage project Together.org [82] has used such tangible models of prehistoric standing stones as interface devices. The models can be positioned on a table-top display to load specific data relevant to the object that is placed on the table. However, when these tangible objects encode data, they are often called physical visualizations.

Physical visualizations have been used for many years. In the late 1950's researchers started to use physical representations of molecules [59] that developed into the so-called "ball and spoke" models, which are widely used in chemistry and biology education. Some data physicalizations have become works of art in their own right. They are beautiful, but explain and encode underlying data. Jansen and colleagues explain many opportunities for physical data visualizations [54].

Currently there is considerable interest in the use of 3D printed models for presenting graphical information to people who are blind or severely vision impaired. Brown and Hurst [14] describe a tool for automatically generating 3D printed line graphs from equations or tabular data sets, while $\mathrm{Hu}$ [52] investigated $3 \mathrm{D}$ printed bar charts.

An interesting area of current research is to use static tangible models and to project dynamic content. For instance, public art events project dynamically moving video onto the side of buildings, architects have started to project moving imagery onto models and miniatures of buildings, while scientists have projected fluid flow simulation and visualization data onto sand [96]. These tangible visualizations are both static (because they have a fixed tangible content) and dynamic (because moving simulation data, and dynamic data visualizations are augmented on top).

More recently, there has also been promising research into dynamic, tangible visualizations. These works blur the lines between haptic physicalisations and haptic data-visualization devices. Actuators, solenoids or stepper-motors are used to move bars or paddles such to dynamically encode information. For example, McGookin et al. [79] developed a tangible user interface (TUI) that could display line and bar graphs in a form that could be touched, whereas Crider et al. [27] used a tangible mixing-board to control filtered values in a visualization application [27]. The inForm system [35] or the Haptic Edge Display [53] (both from MIT) enable different physicalisations to become dynamic. They allow the computer to represent data in a tactile way, and enable the user to feel the data as the input data values change.

Haptic Devices. Different devices have been created to recreate forces, vibrations, textures, temperature changes or even induce pain. Much like with the display of graphical cues, whereby the user understands that an object is three dimensional because it has a shadow and has light and dark side, haptic cues enable the user perceive shape. A force applied to a finger could represent an edge of an object, a vibration could present an event has happened, etc. Haptic interaction then may occur directly, such when the user directly touches the device with their finger, or indirectly through the use of a tool (such as using a pencil or scissors). 
Haptic research has a long history. Early researchers created remote teleoperation devices, often used in dangerous or remote locations (such as the 1950s Argonne National Laboratory device to remotely handle radioactive material); researchers in the late 1990s invented many new haptic devices that were small and used by researchers in academic institutions (such as the popular Phantom force feedback device [77]). The 2000s saw widespread commercialisation and use of these small devices (such as vibrotactile devices in mobile phones and game controllers).

Due to the interest and widespread potential use of haptics, a number or survey papers have been published. These cover devices, techniques and applications (see, for example, $[42,75,89])$. Hayward and MacLean [42] recognise four methods for creating haptic sensations: vibrotactile devices, force-feedback systems, surface displays, and distributed tactile displays. In their review, they describe the means to construct experimental devices and the software components needed to drive them. In their companion paper [75] they address the problem of designing interaction with such devices, but also discuss the role of haptics in "offering an additional communication conduit, providing we recognize the importance of attentional design and the overall user environment and its loading". Finally, Panéels and Roberts [89] comprehensively survey the use of haptics in data visualization.

\subsubsection{Tactile and Kinaesthetic Technologies}

To simplify the haptic sensory field many researchers divide the domain of haptics into two categories: tactile (or cutaneous) and kinaesthetic cues. This is a convenient simplification and can be used to classify much of the research within the haptics domain. Moreover, many of the developed technologies either elicit small movements and excite the receptors in our skin, or provide large forces that change human muscle and limb positions. Consequently we focus on cutaneous and kinaesthetic display technology here.

Cutaneous devices. Imagine holding a glass of hot water. Through our fingertips we notice the temperature of the glass (and therefore conclude the temperature of the water), along with the texture and smoothness of the glass. We might also understand the frictional force of the glass (by understanding how much we need to grip the glass with our fingers, such that it will not fall out of our hands). We can also feel the force applied by gravity, and maybe understand how the water moves or vibrates. These are therefore some of our haptic variables that we have the potential to use, and display data therein. Others include: temperature [56], contact geometry [22], slippage [16], and vibration [103].

The popularity of vibrotactile devices has certainly been helped by the growth of mobile and smart technologies. Every mobile phone has a vibrotactile device that vibrates when the phone rings, or vibrates when the user touches the display to press a button. Vibration devices can also be used to assist navigation (such as mimicking bumps and collisions in games) and providing cues that allow the 
user to infer about an object location or direction to be followed. Vibrotactile devices have also been woven into clothing, from a tactile array sleeve [11] to gloves [85].

A well-known application area for cutaneous haptic technologies is to present data for blind or severely vision-impaired users. A common technique is to use tactile graphics, which are raised line drawings shown on some kind of tactile display. Vidal-Verdu and Hafez [116] present a survey of different kinds tactile displays categorising them as either refreshable or non-refreshable. Refreshable displays use actuators that dynamically change the object, while non-refreshable displays are static, such as produced by embossing with raised dots using a Braille embosser, printing onto swell paper and thermoforming.

Researchers are starting to explore and develop technology that presents other forms of cutaneous response. Devices such as T-pad [120] or TeslaTouch [6] enable friction to be created through electrovibration. These devices create electrostatic friction that allow different frictional values to be represented to the user. Several surveys on tactile devices and techniques $[8,111]$ present different examples and allow understanding the evolution of this technology.

A recent technology that shows huge promise for dynamic visualizations, is airborne haptics. Projects such as the noncontact tactile display [50] and UltraHaptics [20] provide a way for mid-air forces to be felt on (say) the hand of the user. While the forces that have been created by airborne haptics are currently very low, they have huge potential to change the way we feel and interact with three-dimensional environments.

Kinaesthetic devices. Recalling our previous example of holding a glass of water, we can perceive its weight and the hardness of the material it is made from through our sense of touch. Instinctively, we apply a force that is capable of holding the glass without deforming it. If someone slides an object on a table and we have to grab it, the force we have to apply must be enough to stop it: we would somehow feel the inertia that is moving that object in our direction.

Kinaesthetic devices have been used for many years usually to provide force feedback for users while they are interacting with virtual representations of physical objects. These devices allow sensing the object's weight, hardness and inertia [16]. This resistance to users' action can be used to encode data, and as such weight, stiffness and inertia are our haptic variables in this modality.

Force feedback devices have been largely used in simulators to convey intrinsic information regarding the object or phenomena the user is manipulating or studying. For example, in medical simulators, force-feedback devices have been employed for training students in different tasks from simple palpation with a virtual finger [21] and needle insertion [44] to minimally invasive surgeries $[5,43]$. Coles et al. [25] present a thorough survey of the use of haptic devices in medical training applications, giving many examples of commercial and experimental systems, many of them employing immersive technologies. The use of haptics in medical training applications is in continuous development and mostly taskspecific, such as the force-feedback devices developed by Dargar et al. [29]. Among 
other application domains that benefit from such techniques we can cite driver assistance systems [90] and haptic exploration of computational fluids [23].

Since these applications are intended to provide kinaesthetic sensations about objects or phenomena, they map the properties of objects to those on the device. The accuracy of such mappings is essential for a correct analysis. Recent work on data-driven haptic rendering [122] aims at modelling and rendering both stiffness and friction of a deformable object, by providing frictional slip interaction within sliding exploration on a large surface area on an inhomogeneous deformable object, with a perceptually acceptable accuracy level. The authors claim their system is among the first to support fully unconstrained exploration of deformable objects including rubbing, poking, and stroking, with reasonable accuracy.

\subsubsection{Haptics in Immersive Analytics}

There are many opportunities to more effectively use haptics in immersive analytics. The first obvious way is to physically move the human in the world. In the large, solutions such as cable robots can place the human on a chair in the centre of a room, and as the chair is physically moved in the room, the user moves in the data space. Through this technology users can feel immersed in the centre of their data. Other solutions, such as treadmills or walking platforms, help to sense walking motions, but keep the user in a static location. In fact, one challenge for immersive visualization is that the user is often physically located in a relatively small space, making it difficult for the VR system to track the user over the long distances that would be useful to mimic very large virtual spaces. Redirected walking [95] is one solution where the visual simulation tricks the users into believing that they are walking in a straight line when in fact they are walking (for instance) in a circle.

Haptic wearables or even a full-body haptic interaction suit would also be useful in immersive analytics. Many of these suits and wearables use vibrotactile devices to present a haptic response. Forces are possible, through using exoskeleton devices. Shull and Damian [109] provide a review of haptic wearables and Bogue [12] reviews exoskeleton and robotic prosthetics.

Another possibility of providing a sense of immersion in a data space coupled with analytics features could be achieved by combining vibrotactile, head-mounted displays with dynamic tangible objects. Vibrotactile head-mounted displays allow a more accurate, precise, and faster target localization in an active head pointing task [55], and could be used to locate points of interest in the data space. Dynamic tangible devices such as the Haptic Edge Display [53] and the Emergeables [101] allow investigation of interesting interactive techniques that could be used to analyze data subsets. As mentioned before, the Haptic Edge Display [53] represents data through actuated pins placed on the side of a mobile display, which can be sensed and manipulated. Emergeables [101] is based on the concept of creating a physical (deformable) surface that displays data and presents physical controls like buttons and sliders. More interesting, these controls emerge from the display when needed and disappear back into the surface when they are no longer needed. The inForm system [35] could also be used to allow 
sensing and interacting with a specific subset of data points located in a (virtual) surrounding space.

\subsection{Smell/Olfaction, Taste/Gustation}

The sense of smell and taste are connected. Smell is a chemical reaction where odorant molecules bind to the olfactory receptors in the nose. Our tongue can distinguish five distinct qualities, whereas the nose can discern hundreds of different substances. Smell and taste receptors combine together to give us the sense of flavour. Smell is a perceptual phenomenon which depends not only on the odorant molecules but the environment and the person [1]. Smells are linked closely to memories, and through perceiving a smell we can recall specific situations or events. Smell also conjures a vast range of emotions. Smells also act subconsciously, and may effect the way we choose our partners. Smells are also used by the body to detect dangers, such as smoke or the smell of rotten food.

\subsubsection{Suitability of Olfaction and Gustation Senses for Immersive Analytics}

Smells are everywhere in the real world: for example, we notice smells of coffee from a room next to ours, or we smell the perfume of someone who had long since walked down the corridor. Thus, using smells in immersive analytics may help the user to believe that a virtual world is more natural or realistic. But how can smell be used to display data? It is easy to imagine that stronger smells could be used to represent larger values in our dataset, or that sweet or pungent smells be used to present categorical data. However, the dimensions of smell perception are not well understood. Zarzo and Stanton [123] provide a review of different odour maps, and Koulakov [65] provide a (MDS) scaling of the dimensions as a picture, with four main segments, as follows:

- sickening, putrid, sharp, pungent, acid, heavy, fatty sour, etc.

- burnt, smoky, nutty, woody, peanut butter, warm, dry, etc.

- fragrant, sweet, floral, light, rose, etc.

- chemical, medical, disinfectant, gasoline, solvent, cool, paint, etc.

Smell and taste vocabulary is an interesting area because often, when faced with some data, a user may need to express what they have discovered. For visualization, a user would be able to express that a value is larger, or smaller, based on the difference in length of a bar on a barchart, for example. The user readily understands the vocabulary of visualization because they are used to plots and pictures, and the (visual) words are more common in every-day language. However, with smell and taste, users are less likely to understand the vocabulary, which is less familiar. Wilson and Stevenson write "the vocabulary of olfaction almost invariably ties the odour to its physical source, e.g., orange or coffee or cheese odours. This is distinctly different than, for example, the vocabulary for color, in which blue, yellow, and red can be distinct percepts in themselves, separate from whatever object produces those reflected wavelengths" [119]. 


\subsubsection{Smell and Taste Technologies}

One of the main problems is to understand the make-up of the molecules that create particular smells. The same is true for a given chemical mixing: it is difficult to predict how the created molecule will smell. It is well understood that small volatile molecules diffuse fast over a small distance, while other larger molecules linger longer, but understanding what smell is perceived from a specific chemical is more complex. Sometimes similar molecules produce similar smells, whereas molecules that are mirror-symetric to each other could produce different odour sensations [1].

Therefore, current smell systems use arrays of pre-mixed chemicals and when a specific scent is required the lid of a pot is opened such to allow it to disperse. An example of this type of technology is the Olfactory Display [87] from the Tokyo Institute of Technology that can create a range of different smells using a solenoid valves to control which canister is open. Some commercial tools have been created to deliver scent, including DigiScents iSmell, ScentWave, and other

devices that have scents on a USB stick to deliver perfumes where you are working. However, each of these technologies have limited use for immersive visualization because they only deliver a few different smells.

One of the challenges with smell is that it disperses in the room, and that the odour can stay for a long time. This means that it is difficult to represent rapidly changing values through the sense of smell. One of the possible solutions is to deliver smell as close to the user's nose. A tube could be run close (or even up) the user's nose. Subsequently, a smaller dose of scent would be needed to be distinguished by the user. And a fan could be used to readily disperse the smell away from the user, to enable more data to be represented. It could be easy to imagine that someone wearing a Head Mounted Display could also receive odours. The sense of smell has been used as a diagnostic tool for physicians for centuries. Recently research has begun to explore how technology can be used to identify and classify odours $[86,114]$. Many olfactory stimulants carry a strong associative memory with a particular place or event, so in addition to their potential for analytic mapping, they can be used to make a particular multisensory immersion more memorable.

\subsection{Designing Multisensorial Immersive Systems}

As humans evolved in a multi-sensorial environment, our perception has learned to adapt, to optimize, and to work with multiple simultaneous stimuli. A common example is perceiving food taste and recognizing food. As the tongue has only a very limited number of different sensors for different tastes, most of our perceived taste requires olfactory information. Our senses work together, delivering information and a more rich picture about the environment we are currently in.

Immersive environments can employ multiple sensorial channels to communicate information. Such information in multisensorial systems can be information about the data, about the state of the system, or about external issues that the 
immersed user is deprived to perceive (e.g., visual perception of the real world in VR). Multisensorial systems may be able to increase the degree of immersion and help users stay focused and maintain a state of flow.

Building multisensorial systems comes with a number of challenges regarding the components involved in such a system - human, computer machinery, display and input technology - and their respective relationships and combinations. In this section, we discuss questions and challenges related to the design of such multi-sensorial immersive systems with a focus on the combination of multiple sensorial channels for communicating information. We first describe how stimuli from multiple sensory sources can be combined to represent data values. Then, we describe capabilities and limitations of the the human perceptual and cognitive system with respect to multiple sensorial stimuli. Finally, we discuss further implications and conditions such as context and training.

\subsubsection{Multi-Sensorial Representations}

In visualization, there are established visual representations for specific purposes and data sets such as node-link diagrams, scatterplots, parallel coordinates, tree maps, Euler diagrams, and many others [84].

Such representations employ lower level components (e.g., visual marks [9]), their sensorial attributes (e.g., visual variables [9]), as well as rules for placing elements on a 2D space (e.g., force-directed layout, axis values on a scatterplot). Representations can be learned and applied to different problems (data), or serve as blueprints and being modified and extended. Some representations support specific tasks; a node-link diagram supports path following tasks in networks, while adjacency matrices support cluster detection; scatterplots allow guessing about the correlation between two variables (dimensions), but parallel coordinate plots show more dimensions simultaneously.

In multi-sensorial systems the concept of the visual representation must be re-conceptualised into a sensorial representation and the combination of multiple sensorial representations: a multi-sensorial representation. As defined previously, a sensorial representation results from a sensorial mapping and as such, it can involve a variety of sensorial channels.

Some questions arise from this definition: What is a \{sensorial, auditory, olfactorial, haptic, etc.\} representation? Are there specific recurring design patterns for auditory or haptic communication suitable for data representations? What are the correspondents to visual element and layout in the non-visual channels? What is the equivalent of a node-link diagram or barchart in haptics or audition?

At first, existing representations (in some sensorial channel) can serve as basis to build multi-sensorial systems by augmenting this base representation through other channels.

This leads to two questions in combining multi-sensorial representations:

1. Which individual sensorial representations does my system (need to) support?, and 
2. How to combine these sensorial representations into a consistent user interface experience?

Answering the first question refers to the variety of external and empirical factors: which technology is available to the interface designer and end user, how effective are individual sensorial channels, how real should the sensorial mappings be, e.g., representing olfactorial data through olfactorial stimuli, or affective issues such as the wish to represent specific values through silent and others through loud sounds in order to provoke a feeling of contrast or even physical discomfort.

The second question about how to combine multiple sensorial representations into a consistent user interface experience is certainly much less constrained by external factors, but tied to individual and technological factors. There may be several modes in which combinations can happen, such as

- redundant or complementary

- synchronous or asynchronous

- permanent or on-demand

- and certainly others.

For example, visualization designers can decide to redundantly encode the same data value by two complementary visual variables. Redundant encoding aims to increase the precision with which users can decode the visualization, as two stimuli encode the same value. Redundant encoding in multisensorial systems means to combine stimuli across sensorial channels, for example, colour and sound pitch can both be used to encode the same data value. There could even be three or more redundant mappings for the same value, eventually including all senses. Moreover, the sound can be played on-demand only when the user hovers a data point in visual representation or when he/she touches an element in a physical visualization. Here, the sound can even encode additional information about the touched/hovered data object. Eventually, sound or olfaction can be permanent ambient stimuli giving some background information about the data.

For example, haptic representation is often augmented with audio. The TTT (Talking Tactile Tablet) [71] uses a printed tactile graphic on top of a pressuresensitive touchpad, and an appropriate audio file is played when an object is touched. In MultiVis [80] the authors used a force-feedback device and non-speech audio to provide quick overviews of bar charts while Petrie et al. [91] used a mixture of sound and haptic guidance with a force-feedback joystick to present UML diagrams to blind software engineers. More recently, GraVVITAS [38] uses a combination of audio and vibratory feedback to present a variety of information graphics on a touch screen without the need for printed tactile overlays.

\subsubsection{Leveraging Human Perceptual Abilities}

Differentiation. When mapping data to sensorial channels and stimuli, some senses may perform better depending on the data attribute to be represented as 
well as the task at hand. Visual perception is definitely one of the most important factors of human perception concerning to immersive visualization/analytics; mappings from data elements to visual elements, such as colour, shape, position, texture, and transparency have been well discussed $[78,115]$ to achieve effective visualization. Similarly, data sonification can be studies by considering relationships between acoustic features [37], such as loudness, frequency, tone, and human perception. Impression and emotion are well associated with visual/acoustic expressions. For example, Hervner [48] proposed eight groups of words to express the impressions and emotions evoked by music. In other words, it is important to appropriately select the diverse, sensorial representations to realize impressive and emotional immersive analytics.

Haptic devices will be often useful to operate and control immersive analytics systems. However, effectiveness, safety and comfort of immersive analytics will depend on how we consider ability of perceiving and tolerating haptic features such as temperature, texture, vibration, weight, hardness, inertia, and even pain. Sense of smell and taste are also important to realize impressive and realistic immersive analytics. Technologies for these sense are expected to be applied to immersive analytics systems.

Focus and Training. Humans can focus on specific sensorial stimuli (focused attention) as well as train their sensitivity for a specific visual channel. For example, we can focus on shapes or colours in visual search while ignoring shapes or positions of objects. There are cases where a stimulus in one channel can break this attention and force the brain to pay attention to this stimulus. Common examples include a loud sound, a sharp smell, or a moving visual object.

The brain can also be trained to detect dissonances, position, and distance in sound through shorter or livelong learning processes, like what happen with musicians, artists, dancers, and parfumiers. Besides training for profession, disabled persons often develop specific skills in certain senses. Examples include fine haptic differences and the learning of complex haptic patterns such as in Braille as well as determining the location where a sound comes from.

In the contrary case, we try to avoid any stimuli, when, for example, we try to sleep or concentrate on a demanding task. In a way, our brain is constantly processing input to deliver information and create a reality around us.

However, there are cases where such multimodal perception can be tricked and misleading, especially in virtual environments where almost each stimulus has an artificial origin.

\subsubsection{Perceptual and Cognitive Challenges}

Human perception is impressive but far from perfect - in an absolute sense. Besides natural limitations such as visible and auditory spectra, we may have limitations on our perception due to some impairment from birth, ageing, or accident. It is important to discuss how to consider any perceptual limitation so that we can develop immersive analytics systems able to be used by wide range of users. 
Individual sensorial channels. In terms of visual representation, object arrangements and their relative positions is very important. This is especially true in $3 \mathrm{D}$ environment with stereoscopic visualization. Visual occlusion, the way that objects are tangled or shaded, gives a powerful visual clue with the visual perception of the depth ordering. This information is not the same than the depth perception that can be retrieved from stereoscopic visualization. The occlusion can work by itself on 2D screen to perceive the depth ordering, but in $3 \mathrm{D}$ environment it is crucial that both occlusion and depth represent the same information. If this is not the case, the user will be confused and disoriented. This triggers potential complex problem when dealing with transparency and colour blending [117]. The Stroop effect [112] demonstrates an interference effect between the text we read and its visual colour. In terms of cognitive workload, it is hard to read the name of a written colour than to say its colour. This effects shows how colour perception overtake the reading process, and such phenomena is widely used to create psychological test (e.g., double tasks). Further limitations for vision result from diseases and defects: myopia, astigmatism, presbyopia, and colour-blindness.

Sound that reaches our ear has naturally been distorted by the environment and its perceptual capability, such as through distance (sounds getting quieter), masking (loud sounds mask quite sounds), or echo. The making effect has been successfully turned into an asset to store and compress sound in the MP3 audio compression. Roger Shepard blends between two different sounds to create a musical scale, which can seem to increase in pitch forever [107]. In the same way, Jean-Claude Risset extended this to rhythm perception where the tempo never stops to decrease [98]. Eventually, people suffer from natural hearing loss due to age or over-exposure.

Contradicting stimuli. In the simplest case, stimuli from two different senses contradict each other; we smell a certain food but consistency or even taste are not as expected; we see but do not hear the electric car on the street; we hear but do not see the airplane in the cloudy sky; etc. Another example which considerable consequences in virtual environments are visual stimuli contradicting our sense of balance and orientation; an environment that visually is moving but not physically can cause motion sickess. The inverse, an environment that physically is moving but not visually, causes problems for some people on ships and on curvy car tracks.

Moreover, it is not only the actual senses that make up reality, but also knowledge about the world and about what to expect and what not. For example, seeing an object with a realistic stone texture lets us assume it is heavy and we are surprised if lifting it is easy (in fact, our brain unconsciously prepares our muscles to lift an object of that perceived size and weight).

Sensory crosstalk. Multisensory stimuli can lead to the perception of false and non-existent stimuli, as the brain is constantly interchanging signals between brain regions responsible for processing stimuli from different senses [17, 51]. 
Sensory-crosstalk, a term used by Howes [51] can range from two stimuli being truly equivalent in their information, over an enhancement, to eventually the emergence of information.

For example, in the McGurk effect [81] the visual sense interferes with the auditorial sense creating wrong auditory information; the same sound (e.g., $b a$ ) while watching lip movement for another sounds (e.g., ga) becomes a third sound in the brain (in this case $d a$ ). The other way is possible, too, as shown by Shams et al. [106]; the frequency of a visual signal can be tricked by the frequency of a "synchronized" audio signal. ${ }^{2}$ Shams et al. synchronized the single flashing of a dot with two and more auditory beep-signals, leading study participants to perceive two and more visual flashes.

In multisensorial systems, such effects can eventually lead to the falsification of signals. However, which respective cases do enhance or falsify has been little investigated so far.

We have to be careful not only about the way in which stimuli from different sources are being combined, but even simpler, about their number. Again, more stimuli (more information) can improve our brain in perceiving and deciding for the "the right" information but too much information can result in the contrary: perceptual and cognitive overload leading to ignoring stimuli or problems in deciding with stimuli to trust in cases where stimuli are contradicting each other.

Perceptual overload. Perceptual or sensorial overload occurs when the brain is confronted with too many stimuli from the same or different channels and possibly over a longer time. Perceptual overload requires the brain to filter and concentrate on a subset of stimuli. While this can lead to selective attention, in other cases perceptual overload can lead to missing information and in the worst case to stress-like symptoms and a temporary decrease in cognitive abilities. The case is well known from visualization where too many visual elements with different visual encodings reduce an observers ability to correctly decode information; cluttered node-link diagrams and parallel coordinates plots, maps and scatterplots with too many visual variables; inappropriate use of colour, animation, and animated visual variables such as flickering, in general. The same principles hold for the other channels; too many tones, too many combination of aural variables, and so forth.

In any case, the purpose of any system with the goal of communicating information through multiple senses is to carefully select the channels best suited for some information and to carefully combine them. One goal could be to reduce a possible sensorial overload in one sense by distributing information over several senses.

Cognitive overload. Similar to sensorial overload, and a possible consequence of sensorial overload is cognitive overload. While sensorial overload is specific to one or multiple senses simultaneously, and describes an overload for what and

\footnotetext{
${ }^{2}$ https://www.youtube.com/watch?v=D3Z1cxA2Tp0
} 
how things are perceived, cognitive (or information) overload usually refers to processing an abundance of perceived information [62]. Cognitive overload can be caused by switching tasks rapidly (though not taking breaks), multi-tasking, distraction and interruption [41]. As Miller writes, the human brain is able to keep between 5-9 "items" in memory simultaneously [83]. Interruptions and sudden changes in sensorial input can influence the current state of the brain in a way that makes us forget what we have been just thinking $[62,83]$. In multi-sensorial information systems this can result from signals interrupting the user in the task s/he is currently focusing on (e.g., an aural notification that some data has been updated).

The challenge in multi-sensorial information systems is to support a users mental state of flow, to minimize distraction and to adapt all, or at least the majority of sensorial stimuli to the task the user is currently performing. For example, stimuli signalling system states should be muted according to nonrelevance to the current task; stimuli from different sources should focus on the current user tasks and deliver a holistic picture of information; stimuli should complement each other or encode information redundantly.

Pre-knowledge and Training. As occurs with many computational systems, users of immersive analytic applications may have a wide range of knowledge and experience levels. Besides knowledge on the application domain, users also may have a wide range of skills and experiences of the underlying technologies used in visual analytics, virtual/augmented reality, and human-computer interaction. One may need to consider these differences for developing useful immersive analytics systems. For example, users may need to train 3D recognition skills such as spatial awareness or shape understanding for using spatially immersive environments. Also, 3D operations skills using walkthrough interfaces and haptic devices may be required. If the application demands creative or artistic activities during analytics processes, users may need to train immersive-specific skills. For example, 3D modeling and music playing are typical activities which users may need to train for using immersive environments.

\subsubsection{Context and Environment}

We may need to consider the context of applications and runtime environments while designing immersive analytics systems. Typical factors that we may need to consider are task models, including collaborative tasks, and the physical environment where they are to be used.

Task models and collaborative tasks. Visualization research communities have a history of defining task models. We can start from the information visualization mantra by Shneiderman [108] and the visual analytics mantra by Keim et al. [58], both simply defining repetitive tasks. The sense-making loop is well defined for visual analytics [57], being a good reference for system design of immersive analytics tools. 
As occurs with many virtual reality applications, immersive analytics may also be useful for collaborative tasks, which assume that multiple users are immersed into the same (virtual) analytics space and collaboratively work there. Typical applications where collaborative tasks in immersive environments can be borrowed from many virtual reality application domains:

- Physical space simulation and analysis for various academic and industrial fields such as fluid dynamics and bioinformatics.

- Industrial design, where designers, engineers, salesmen and employers may work collaboratively in immersive environments.

- Transportation/disaster/security analysis, where experts from different fields of domain may use immersive analytics.

User Environment: Immersive analytics systems are often built on top of existing virtual/augmented reality environments. There are several surveys discussing what kinds of technologies have evolved from virtual/augmented reality systems $[2,70]$. A number of typical environments and technologies exist, which we may need to be appropriately select for developing immersive analytics systems.

- Diverse computing environments: large-scale, personal, or mobile computers can be used for immersive analytics.

- Display systems: stereoscopic or head-mounted displays are often mandatory for immersive system development. The size of displays is also an important factor for system design.

- Speakers systems: surround audio systems or head-phones are often mandatory for immersive acoustic systems.

- Input devices for navigation, pointing, and gesture.

- Haptic technologies for sensing forces, temperature, vibration, and other stimuli.

In some cases, no visual output may be available while doing other tasks. For example, it is difficult to carefully look at the display of car navigation systems during driving a car. We also need to consider that no- or one-hand operation may be only allowed while doing other tasks. Again, alternative sensory is desirable for such situations. In other cases, we are often not allowed to use input/output sounds or perform large gestures during immersive analytics, for example in public spaces. Alternative sensory is desirable to make the system suitable for use under such situations.

\subsection{Discussion and Research Challenges}

Auditory interfaces: To date, sound has been largely underutilized in multisensory immersive analytic applications. Just as visual representations require good visual design skills, auditory interfaces require good sonic design skills (a topic not as widely taught as visual design). Moreover, many environments are not ideal for critical listening, due to ambient noise, room coloration or reverberation, 
for example. Recent HMD devices have included stereo headphones which can help minimise some of these problems. What remains an important research challenge is in the successful mapping of data variables to sonic variables. Unlike visualization, few conventions exist, so the user must necessarily learn mappings anew. Spatialization and immersion are two important features that sound can effectively exploit; the challenge is how to integrate sound into multisensory systems so that the auditory components work in harmony with the other sensory stimuli effectively.

Haptic interfaces: Haptic technologies have huge potential for integration in immersive analytics. We can readily consider three sizes of devices. First, there is huge potential for large-scale physical dynamic devices that control the whole body of a user. For example, treadmills, chairs or even small rooms can be vibrated, moved and rotated. These provide physical sensations that enable the users to move and position themselves directly inside large scale data space. But these devices are typically expensive and require careful health and safety controls. Second, wearable technologies are developing fast and have many uses for immersive analytics. This has been aided by the pervasiveness of cheap and robust vibrotactile devices and the acceptability of wearable devices and clothes. There are many research challenges, and opportunities. While several companies have tried to build haptic suits they have not been currently successful. There is a need to develop modular systems that are robust and cost effective. Third, there are important opportunities for high-fidelity dynamic tactile devices. Currently there are several device types that are being explored in research laboratories, from friction-based displays and vibro-tactile head mounted displays to airborne haptics. However, each of these technologies need to mature further and become cheaper. Finally, there are huge opportunities to integrate tangible objects into virtual worlds and use them for immersive analytics. While three dimensional printing is cheap, and is becoming widely used, there are few middleware libraries or applications that utilise them effectively.

Olfactorial interfaces: Technologies to integrate smell and taste in an immersive analytics system are probably the least mature of all the senses. Certainly, because of the complexity of how humans perceive smell, it is impossible to create and mix smells dynamically. Therefore current technological solutions issue pre-canned odours on demand. Another challenge for immersive analytics is how to deliver the appropriate smells to the user. Odours linger in the room, and need to be flushed out by neutral odours, it would be better to deliver smaller quantities of odour directly to participants, through a personal delivery system. However this can be intrusive and would need the user to wear some kind of nose-mounted display.

Multisensorial representations: Combining sensorial data representation is still an open field where there are many opportunities worth to be explored to leverage user data perception. For instance, sensorial prioritisation triggers numerous questions. While visualization has the largest bandwidth in terms of data communication power, little is known regarding how interruption is prioritized by the human perception system within multi-modal senses. More 
open questions are concerned with how to create effective combinations. The purpose of a combination may be exploratory, analytical, aiming at presentation and "telling stories about data" or creating a sense of immersion, presence, and experience. Further chapters discuss other issues in combining different sensorial data representations.

Interaction: Interaction in multisensorial systems will most likely happen through haptics, e.g., pressing buttons, touching surfaces, grabbing and reaching to objects, etc. However, there are already cases that involve speech (e. g. Microsoft Hololens). More futuristic scenarios envision human-brain interaction (BCI). BCI is a communication pathway between human brain and an external device. Such techniques are usually envisaged for impaired users but they open promising opportunities to enhance user cognitive perception with a direct and more controlled communication channel.

\subsection{Conclusion}

Multisensory immersive analytics is an exciting frontier and natural extension of research in visualization and visual analytics. As we have outlined in this chapter, the potential applications are widespread across many different disciplines and tasks. While we have shown a number of examples that demonstrate existing achievements in this field, there remains many important challenges in order for the multisensory experience to reach its full potential (see [69] for examples in sonification).

Other issues include how to map data to multisensory channels and how interaction is interwoven with those sensory channels. We also need to more effectively create data representations tailored to emerging multisensory display technologies. As the capability of technology to realise more immersive multisensorial experiences becomes more prevalent, the impact and application for more fully understanding data will be achieved.

\section{References}

1. Auffarth, B.: Understanding smell - the olfactory stimulus problem. Neuroscience \& Biobehavioral Reviews 37(8), 1667-1679 (2013)

2. Azuma, R.T.: A survey of augmented reality. Presence: Teleoperators and Virtual Environments 6(4), 355-385 (1997)

3. Ball, R., North, C., Bowman, D.A.: Move to improve: Promoting physical navigation to increase user performance with large displays. In: Proceedings of the SIGCHI Conference on Human Factors in Computing Systems. pp. 191-200. CHI '07, ACM (2007)

4. Barrett, N.: Interactive spatial sonification of multidimensional data for composition and auditory display. Computer Music Journal (2016)

5. Basdogan, C., De, S., Kim, J., Muniyandi, M., Kim, H., Srinivasan, M.A.: Haptics in minimally invasive surgical simulation and training. IEEE Computer Graphics and Applications 24(2), 56-64 (2004) 
6. Bau, O., Poupyrev, I., Israr, A., Harrison, C.: Teslatouch: electrovibration for touch surfaces. In: Proceedings of the 23nd Annual ACM Symposium on User Interface Software and Technology. pp. 283-292. ACM (2010)

7. Baum, G., Gotsis, M., Chang, C., Drinkwater, R., Clair, D.S.: Synthecology: Sound use of audio in teleimmersion. In: Proceedings Stereoscopic Displays and Virtual Reality Systems XIII. vol. 6055. SPIE the Engineering Reality of Virtual Reality (2006)

8. Benali-khoudja, M., Hafez, M., marc Alex, J., Kheddar, A.: Tactile interfaces: a state-of-the-art survey. In: Int. Symposium on Robotics. pp. 721-726 (2004)

9. Bertin, J.: Sémiologie graphique: Les diagrammes - Les réseaux - Les cartes. Editions de l'Ecole Hautes Etudes en Sciences, Paris, France, les réimpressions edn. (1967)

10. Bezerianos, A., Isenberg, P.: Perception of visual variables on tiled wall-sized displays for information visualization applications. IEEE Transactions on Visualization and Computer Graphics 18(12), 2516-2525 (2012)

11. Bloomfield, A., Badler, N.I.: Virtual training via vibrotactile arrays. Presence: Teleoperators and Virtual Environments 17(2), 103-120 (2008)

12. Bogue, R.: Exoskeletons and robotic prosthetics: a review of recent developments. Industrial Robot: An International Journal 36(5), 421-427 (2009)

13. Bowman, D.A., Kruijff, E., LaViola, J., Poupirev, I.: User Interfaces - Theory and Practice. Addison Wesley, Boston, USA (2005)

14. Brown, C., Hurst, A.: Viztouch: automatically generated tactile visualizations of coordinate spaces. In: Proceedings of the Sixth International Conference on Tangible, Embedded and Embodied Interaction. pp. 131-138. ACM (2012)

15. Brown, L.M., Brewster, S.A., Ramloll, S., Burton, R., Riedel, B.: Design guidelines for audio presentation of graphs and tables (2003)

16. Burdea, G.C.: Force and Touch Feedback for Virtual Reality. John Wiley \& Sons, Inc., New York, NY, USA (1996)

17. Calvert, G., Spence, C., Stein, B.E.: The Handbook of Multisensory Processes. MIT Press (2004)

18. Card, S.K., Mackinlay, J.D., Shneiderman, B. (eds.): Readings in Information Visualization: Using Vision to Think. Morgan Kaufmann Publishers, San Francisco (1999)

19. Carpendale, M.: Considering visual variables as a basis for information visualisation (2003)

20. Carter, T., Seah, S.A., Long, B., Drinkwater, B., Subramanian, S.: Ultrahaptics: multi-point mid-air haptic feedback for touch surfaces. In: Proceedings of the 26 th Annual ACM Symposium on User Interface Software and Technology. pp. 505-514. ACM (2013)

21. Chen, H., Wu, W., Sun, H., Heng, P.A.: Dynamic touch-enabled virtual palpation. Computer Animation and Virtual Worlds 18(4-5) (2007)

22. Cini, G., Frisoli, A., Marcheschi, S., Salsedo, F., Bergamasco, M.: A novel fingertip haptic device for display of local contact geometry. In: Proceedings of the First Joint Eurohaptics Conference and Symposium on Haptic Interfaces for Virtual Environment and Teleoperator Systems. pp. 602-605. IEEE Computer Society (2005)

23. Cirio, G., Marchal, M., Hillaire, S., Lecuyer, A.: Six degrees-of-freedom haptic interaction with fluids. IEEE Transactions on Visualization and Computer Graphics 17(11), 1714-1727 (2011)

24. Cleveland, W.S., McGill, R.: Graphical perception: Theory, experimentation and application to the development of graphical methods. Journal of the American Statistical Association 79(387), 531-554 (1984) 
25. Coles, T.R., Meglan, D., John, N.W.: The role of haptics in medical training simulators: A survey of the state of the art. IEEE Transactions on Haptics 4(1), 51-66 (2011)

26. Cordeil, M., Dwyer, T., Hurter, C.: Immersive solutions for future air traffic control and management. In: Proceedings of the 2016 ACM Companion on Interactive Surfaces and Spaces. pp. 25-31. ISS Companion '16, ACM, New York, NY, USA (2016)

27. Crider, M., Bergner, S., Smyth, T.N., Möller, T., Tory, M.K., Kirkpatrick, A.E., Weiskopf, D.: A mixing board interface for graphics and visualization applications. In: Proceedings of Graphics Interface. pp. 87-94 (2007)

28. Cunningham, J.P., Ghahramani, Z.: Linear Dimensionality Reduction: Survey, Insights, and Generalizations (2015)

29. Dargar, S., De, S., Sankaranarayanan, G.: Development of a haptic interface for natural orifice translumenal endoscopic surgery simulation. IEEE Transactions on Haptics 9(3), 333-344 (2016)

30. Dingler, T., Brewster, S., Butz, A.: Audiofeeds - a mobile auditory application for monitoring online activities. In: In Proceedings of ACM Multimedia (Florence, Italy), ACM Press (2010)

31. Engel, D., Hüttenberger, L., Hamann, B.: A survey of dimension reduction methods for high-dimensional data analysis and visualization. In: VLUDS (2011)

32. Few, S. (ed.): Information Dashboard Design: The Effective Visual Communication of Data. Analytics Press (2006)

33. Flowers, J.H., Buhman, D.C., Turnage, K.D.: Cross-modal equivalence of visual and auditory scatterplots for exploring bivariate data samples. Human Factors: The Journal of the Human Factors and Ergonomics Society 39(3), 341-351 (1997)

34. Foley, H., Matlin, M.: Sensation and Perception. Psychology Press (2015)

35. Follmer, S., Leithinger, D., Olwal, A., Hogge, A., Ishii, H.: inFORM: dynamic physical affordances and constraints through shape and object actuation. In: ACM Symposium on User Interface Software and Technology. vol. 13, pp. 417-426 (2013)

36. Franklin, K.M., Roberts, J.C.: Pie chart sonification. In: Proceedings of the Seventh International Conference on Information Visualization. pp. 4-9. IEEE Computer Society, Washington, DC, USA (2003)

37. Gaver, W.W.: What in the world do we hear?: An ecological approach to auditory event perception. Ecological Psychology 5(1), 1-29 (1993)

38. Goncu, C., Marriott, K.: GraVVITAS: generic multi-touch presentation of accessible graphics. In: IFIP Conference on Human-Computer Interaction. pp. 30-48. Springer (2011)

39. Goodwin, A.W., Wheat, H.E.: Physiological mechanisms of the receptor system. In: Grunwald, M. (ed.) Human Haptic Perception: Basics and Applications. pp. 93-102. Birkhäuser Basel (2008)

40. Grey, J.M.: Multidimensional perceptual scaling of musical timbres. Journal of the Acoustical Society of America 61(5), 1270-1277 (1977)

41. Hallowell, E.M.: Overloaded circuits. Harvard business review p. 11 (2005)

42. Hayward, V., Maclean, K.E.: Do it yourself haptics: part i. IEEE Robotics Automation Magazine 14(4), 88-104 (2007)

43. Heng, P.A., Cheng, C.Y., Wong, T.T., Xu, Y., Chui, Y.P., Chan, K.M., Tso, S.K.: A virtual-reality training system for knee arthroscopic surgery. Trans. Info. Tech. Biomed. 8(2), 217-227 (2004)

44. Heng, P.A., Wong, T.T., Yang, R., Chui, Y.P., Xie, Y.M., Leung, K.S., Leung, P.C.: Intelligent inferencing and haptic simulation for chinese acupuncture learning 
and training. IEEE Transactions on Information Technology in Biomedicine 10(1), 28-41 (2006)

45. Hensel, H.: Cutaneous Thermoreceptors. In: Iggo, A. (ed.) Somatosensory System. pp. 79-110. Springer Berlin Heidelberg, Berlin, Heidelberg (1973)

46. Hermann, T., Hunt, A., Neuhoff, J.G.: The Sonification Handbook. Logos Publishing House, Berlin, Germany (2011)

47. Hermann, T.: Taxonomy and definitions for sonification and auditory display. International Community for Auditory Display (2008)

48. Hevner, K.: Experimental studies of the elements of expression in music. The American Journal of Psychology 48(2), 246-268 (1936)

49. Hoggan, E., Brewster, S.: Crosstrainer: Testing the use of multimodal interfaces in situ. In: Proceedings of the ACM Conference on Human Factors in Computing Systems. pp. 333-342. ACM Press (2010)

50. Hoshi, T., Takahashi, M., Iwamoto, T., Shinoda, H.: Noncontact tactile display based on radiation pressure of airborne ultrasound. IEEE Transactions on Haptics 3(3), 155-165 (2010)

51. Howes, D.: Cross-talk between the senses. The Senses and Society 1(3), 381-390 (2006)

52. Hu, M.: Exploring new paradigms for accessible 3D printed graphs. In: Proceedings of the 17th International ACM SIGACCESS Conference on Computers \& Accessibility. pp. 365-366. ACM (2015)

53. Jang, S., Kim, L.H., Tanner, K., Ishii, H., Follmer, S.: Haptic edge display for mobile tactile interaction. In: Proceedings of the 2016 CHI Conference on Human Factors in Computing Systems. pp. 3706-3716. CHI '16 (2016)

54. Jansen, Y., Dragicevic, P., Isenberg, P., Alexander, J., Karnik, A., Kildal, J., Subramanian, S., Hornbæk, K.: Opportunities and challenges for data physicalization. In: Proceedings of the 33rd Annual ACM Conference on Human Factors in Computing Systems. pp. 3227-3236. CHI 15, ACM (2015)

55. de Jesus Oliveira, V.A., Brayda, L., Nedel, L., Maciel, A.: Designing a vibrotactile head-mounted display for spatial awareness in 3D spaces. IEEE Transactions on Visualization and Computer Graphics 23(4), 1409-1417 (2017)

56. Jones, L.A., Berris, M.: The psychophysics of temperature perception and thermalinterface design. In: Proceedings of 10th Symposium on Haptic Interfaces for Virtual Environment and Teleoperator Systems. p. 137. IEEE Computer Society (2002)

57. Keim, D., Andrienko, G., Fekete, J.D., Görg, C., Kohlhammer, J., Melançon, G.: Visual analytics: Definition, process, and challenges. In: Kerren, A., Stasko, J.T., Fekete, J.D., North, C. (eds.) Information Visualization: Human-Centered Issues and Perspectives. pp. 154-175. Springer Berlin Heidelberg, Berlin, Heidelberg (2008)

58. Keim, D.A., Mansmann, F., Schneidewind, J., Thomas, J., Ziegler, H.: Visual analytics: Scope and challenges. Lecture Notes in Computer Science, Visual Data Mining 4404, 76-90 (2008)

59. Kendrew, J.C., Bodo, G., Dintzis, H.M., Parrish, R., Wyckoff, H., Phillips, D.C.: A three-dimensional model of the myoglobin molecule obtained by x-ray analysis. Nature 181(4610), 662-666 (1958)

60. Klapperstueck, M., Czauderna, T., Goncu, C., Glowacki, J., Dwyer, T., Schreiber, F., Marriott, K.: ContextuWall: peer collaboration using (large) displays. In: 2016 Big Data Visual Analytics (BDVA). pp. 1-8 (2016)

61. Klatzky, R.L., Lederman, S.J., Metzger, V.A.: Identifying objects by touch: An "expert system". Perception \& Psychophysics 37(4), 299-302 (1985) 
62. Klingberg, T.: The Overflowing Brain: Information Overload and the Limits of Working Memory. Oxford University Press (2009)

63. Köbben, B., Yaman, M.: Evaluating dynamic visual variables. In: Proceedings of the Seminar on Teaching Animated Cartography, ACI/ICA, Madrid. pp. 45-51 (1996)

64. Kolarik, A.J., Cirstea, S., Pardhan, S., Moore, B.C.: A summary of research investigating echolocation abilities of blind and sighted humans. Hearing Research 310, 60-68 (2014)

65. Koulakov, A.: In search of the structure of human olfactory space. Flavour 3(1), O1 (2014)

66. Kramer, G.: Mapping a single data stream to multiple auditory variables: A subjective approach to creating a compelling design. In: International Conference on Auditory Displays (1996)

67. Kramer, G.: Auditory display: Sonification, audification, and auditory interfaces. Perseus Publishing (1993)

68. Kramer, G., Walker, B., Bonebright, T., Cook, P., Flowers, J., Miner, N., Neuhoff, J.: Sonification report: Status of the field and research agenda (1999)

69. Kramer, G., Walker, B., Bonebright, T., Cook, P., Flowers, J.H., Miner, N., Neuhoff, J.: Sonification report: Status of the field and research agenda (2010)

70. van Krevelen, R., Poelman, R.: A survey of augmented reality: Technologies, applications and limitations. The International Journal of Virtual Reality 9(2), 1-20 (2010)

71. Landau, S., Gourgey, K.: Development of a Talking Tactile Tablet. Information Technology and Disabilities 7(2) (2001)

72. Lederman, S.J., Campbell, J.I.: Tangible graphs for the blind. Human Factors 24(1), 85-100 (1982)

73. Ludovico, L.A., Presti, G.: The sonification space: A reference system for sonification tasks. International Journal of Human-Computer Studies 85, 72-77 (2016)

74. Mackinlay, J.: Automating the design of graphical presentations of relational information. ACM Transactions On Graphics (ToG) 5(2), 110-141 (1986)

75. Maclean, K.E., Hayward, V.: Do it yourself haptics: Part ii [tutorial]. IEEE Robotics Automation Magazine 15(1), 104-119 (2008)

76. Madhyastha, T.M., Reed, D.A.: Data sonification: do you see what I hear? IEEE Software 12(2), 45-56 (1995)

77. Massie, T.H., Salisbury, J.K.: The phantom haptic interface: A device for probing virtual objects. In: Proceedings of the ASME Winter Annual Meeting, Symposium on Haptic Interfaces for Virtual Environment and Teleoperator Systems. vol. 55(1), pp. 295-302 (1994)

78. Mazza, R. (ed.): Introduction to Information Visualization. Springer (2009)

79. McGookin, D., Robertson, E., Brewster, S.: Clutching at straws: Using tangible interaction to provide non-visual access to graphs. In: Proceedings of the ACM Conference on Human Factors in Computing SystemsI. pp. 1715-1724. ACM Press (2010)

80. McGookin, D., Brewster, S.: MultiVis: Improving Access to Visualisations for Visually Impaired People. In: ACM Conference on Human Factors in Computing Systems: Extended Abstracts. pp. 267-270. ACM (2006)

81. McGurk, H., MacDonald, J.: Hearing lips and seeing voices (1976)

82. Miles, H.C., Wilson, A.T., Labrosse, F., Tiddeman, B., Griffiths, S., Edwards, B., Ritsos, P.D., Mearman, J.W., Möller, K., Karl, R., Roberts, J.C.: Alternative representations of 3D-reconstructed heritage data. J. Comput. Cult. Herit. 9(1), 4:1-4:18 (2015) 
83. Miller, G.A.: The magical number seven, plus or minus two: some limits on our capacity for processing information. Psychological Review 63(2), 81 (1956)

84. Munzner, T.: Visualization Analysis and Design. CRC Press (2014)

85. Murray, A.M., Klatzky, R.L., Khosla, P.K.: Psychophysical characterization and testbed validation of a wearable vibrotactile glove for telemanipulation. Presence: Teleoperators and Virtual Environments 12(2), 156-182 (2003)

86. Nakamoto, T., Yosihioka, M., Tanaka, Y., Kobayashi, K., Moriizumi, T., Ueyama, S., Yerazunis, W.: Colorimetric method for odor discrimination using dye-coated plate and multiLED sensor. Sensors and Actuators B: Chemical 116(1-2), 202-206 (2006)

87. Nakamoto, T., Kinoshita, M., Murakami, K., Yossiri, A.: Demonstration of improved olfactory display using rapidly-switching solenoid valves. IEEE Virtual Reality Conference pp. 301-302 (2009)

88. Neuhoff, J.G., Kramer, G., Wayand, J.: Sonification and the interaction of perceptual dimensions: can the data get lost in the map? (2000)

89. Panëels, S., Roberts, J.C.: Review of designs for haptic data visualization. IEEE Transactions on Haptics 3(2), 119-137 (2010)

90. Petermeijer, S.M., Abbink, D.A., Mulder, M., de Winter, J.C.F.: The effect of haptic support systems on driver performance: A literature survey. IEEE Transactions on Haptics 8(4), 467-479 (2015)

91. Petrie, H., Schlieder, C., Blenkhorn, P., Evans, G., King, A., O'Neill, A.M., Ioannidis, G.T., Gallagher, B., Crombie, D., Mager, R., Alafaci, M.: TeDUB: a system for presenting and exploring technical drawings for blind people. In: Miesenberger, K., Klaus, J., Zagler, W. (eds.) International Conference ICCHP 2002, Computers Helping People with Special Needs. pp. 537-539. Springer Berlin Heidelberg, Berlin, Heidelberg (2002)

92. Post, D.L., Greene, E.: Color name boundaries for equally bright stimuli on a CRT: Phase I. Society for Information Display - Digest of Technical Papers 86, 70-73 (1986)

93. Purves, D., Augustine, G.J., Fitzpatrick, D., Hall, W.C., LaMantia, A.S., McNamara, J.O., Williams, S.M. (eds.): Neuroscience: Third Edition. Sinauer Associates, Inc., Sunderland, MA, USA (2004)

94. Ramloll, R., Yu, W., Brewster, S., Riedel, B., Burton, M., Dimigen, G.: Constructing sonified haptic line graphs for the blind student: first steps. In: Proceedings of the Fourth International ACM Conference on Assistive Technologies. pp. 17-25. ACM (2000)

95. Razzaque, S., Swapp, D., Slater, M., Whitton, M.C., Steed, A.: Redirected walking in place. In: Proceedings of the workshop on Virtual environments 2002. pp. 123-130. Eurographics Association (2002)

96. Reed, S., Kreylos, O., Hsi, S., Kellogg, L., Schladow, G., Yikilmaz, M., Segale, H., Silverman, J., Yalowitz, S., Sato, E.: Shaping watersheds exhibit: An interactive, augmented reality sandbox for advancing earth science education. In: AGU Fall Meeting Abstracts. vol. 1, p. 01 (2014)

97. Rincon-Gonzalez, L., Warren, J.P., Meller, D.M., Tillery, S.H.: Haptic interaction of touch and proprioception: Implications for neuroprosthetics. IEEE Transactions on Neural Systems and Rehabilitation Engineering 19(5), 490-500 (2011)

98. Risset, J.C.: Pitch and rhythm paradoxes: Comments on "Auditory paradox based on fractal waveform" [J. Acoust. Soc. Am. 79, 186-189 (1986)]. Acoustical Society of America Journal 80, 961-962 (1986) 
99. Roberts, J.C., Franklin, K.: Haptic glyphs (hlyphs) - structured haptic objects for haptic visualization. In: First Joint Eurohaptics Conference and Symposium on Haptic Interfaces for Virtual Environment and Teleoperator Systems. World Haptics Conference. pp. 369-374 (2005)

100. Robinett, W.: Interactivity and individual viewpoint in shared virtual worlds: The big screen vs. networked personal displays. SIGGRAPH Computer Graphics 28(2), 127-130 (1994)

101. Robinson, S., Coutrix, C., Pearson, J., Rosso, J., Torquato, M.F., Nigay, L., Jones, M.: Emergeables: Deformable displays for continuous eyes-free mobile interaction. In: Proceedings of the 2016 CHI Conference on Human Factors in Computing Systems. pp. 3793-3805. CHI '16, ACM (2016)

102. Rohn, H., Junker, A., Hartmann, A., Grafahrend-Belau, E., Treutler, H., Klapperstück, M., Czauderna, T., Klukas, C., Schreiber, F.: VANTED v2: a framework for systems biology applications. BMC Systems Biology 6(1), 139.1-13 (2012)

103. Salisbury, C., Gillespie, R.B., Tan, H., Barbagli, F., Salisbury, J.K.: Effects of haptic device attributes on vibration detection thresholds. In: Proceedings of the World Haptics 2009 - Third Joint EuroHaptics conference and Symposium on Haptic Interfaces for Virtual Environment and Teleoperator Systems. pp. 115-120. IEEE Computer Society (2009)

104. Saue, S.: A model for interaction in exploratory sonification displays. In: Proceedings of the International Conference on Auditory Display (2000)

105. Scaletti, C., Craig, A.B.: Using sound to extract meaning from complex data. In: Proceedings of SPIE 1459 (Extracting Meaning from Complex Data: Processing, Display, Interaction II). pp. 207-219 (1991)

106. Shams, L., Kamitani, Y., Shimojo, S.: Illusions: What you see is what you hear. Nature 408(6814), 788-788 (2000)

107. Shepard, R.N.: Circularity in judgements of relative pitch. Journal of the Acoustical Society of America 36(12), 2346-2353 (1964)

108. Shneiderman, B.: The eyes have it: A task by data type taxonomy for information visualizations. In: Proceedings of the IEEE Symposium on Visual Languages. pp. 336-343 (1996)

109. Shull, P.B., Damian, D.D.: Haptic wearables as sensory replacement, sensory augmentation and trainer - a review. Journal of NeuroEngineering and Rehabilitation $12(1), 59(2015)$

110. Sounds, S.: Iraq body count. https://soundcloud.com/somatic-sounds/iraq-bodycount-guillaume-potard (2010)

111. Spirkovska, L.: Summary of Tactile User Interfaces Techniques and System. NASA Ames Research Center (2005)

112. Stroop, J.R.: Studies of interference in serial verbal reactions. Journal of Experimental Psychology pp. 643-662 (1935)

113. Takahashi, C., Watt, S.J.: Optimal visual-haptic integration with articulated tools. Experimental Brain Research 235(5), 1361-1373 (2017)

114. Tanaka, Y., Nakamoto, T., Moriizumi, T.: Study of highly sensitive smell sensing system using gas detector tube combined with optical sensor. Sensors and Actuators B: Chemical 119(1), 84-88 (2006)

115. Tory, M., Moller, T.: Human factors in visualization research. IEEE Transactions on Visualization and Computer Graphics 10(1), 72-84 (2004)

116. Vidal-Verdu, F., Hafez, M.: Graphical tactile displays for visually-impaired people. IEEE Transactions on Neural Systems and Rehabilitation Engineering 15(1), 119$130(2007)$ 
117. Ware, C.: Information Visualization: Perception for Design. Morgan Kaufmann Publishers Inc., San Francisco, CA, USA, 3 edn. (2013)

118. Wertheimer, M.: Untersuchungen zur lehre von der gestalt. Psychological Research $1(1), 47-58$ (1922)

119. Wilson, D.A., Stevenson, R.J.: Learning to smell: olfactory perception from neurobiology to behavior. JHU Press (2006)

120. Winfield, L., Glassmire, J., Colgate, J.E., Peshkin, M.: T-pad: Tactile pattern display through variable friction reduction. In: Second Joint EuroHaptics Conference and Symposium on Haptic Interfaces for Virtual Environment and Teleoperator Systems (WHC'07). pp. 421-426. IEEE (2007)

121. Xi, H., Kelley, A.: Sonification of time-series data sets. Bulletin of the American Physical Society 60 (2015)

122. Yim, S., Jeon, S., Choi, S.: Data-driven haptic modeling and rendering of viscoelastic and frictional responses of deformable objects. IEEE Transactions on Haptics 9(4), 548-559 (2016)

123. Zarzo, M., Stanton, D.T.: Understanding the underlying dimensions in perfumers' odor perception space as a basis for developing meaningful odor maps. Attention, Perception, \& Psychophysics 71(2), 225-247 (2009)

124. Zhao, H., Plaisant, C., Shneiderman, B., Lazar, J.: Data sonification for users with visual impairment: A case study with georeferenced data. ACM Transactions on Computer-Human Interaction 15(1) (2008) 